\title{
Determining the Diameter of Reinforcing Bars Inside Concrete Structures Regardless of Magnetic Properties
}

\author{
Alexey V. Mikhailov ${ }^{1, a^{*}}$, Yuri L. Gobov ${ }^{1, b}$ and Leonid V. Mikhailov ${ }^{1, c}$ \\ ${ }^{1}$ M.N. Miheev Institute of Metal Physics of Ural Branch of Russian Academy of Sciences, Russia \\ amikhaylov@imp.uran.ru, bgo@imp.uran.ru, cmikhaylov_lv@imp.uran.ru
}

Keywords: Non-Destructive Testing, Testing of Reinforcement in Concrete, Dipole Model

\begin{abstract}
In this work, using a series of numerical experiments, the dependence of the magnetic field strength of the response of the reinforcing bar to an external magnetizing field on the magnetic properties of the reinforcing bar was investigated. The possibility of determining with high accuracy the diameter of reinforcing bars, regardless of the magnetic properties of the material from which they are made, has been proven.
\end{abstract}

\section{Introduction}

An urgent task in the field of materials processing and materials science is to assess the current state of reinforced concrete structures reinforced with steel reinforcement. The solution to this problem consists in finding the location of the reinforcing mesh, determining the strength characteristics of concrete, detecting delamination of reinforcing bars from concrete, as well as determining such parameters as the depth (thickness of the concrete cover) and the diameter of the bars. It is believed that the strength of concrete after pouring only increases over time, so the current state of the concrete itself can be estimated based on the initial mechanical properties of concrete (concrete grade). For this, a destructive method is used, based on casting samples in the form of cubes, followed by placing them under a press. The value of the critical load at which concrete destruction occurs is an indicator of its grade.

During the operation of reinforced concrete structures, it is necessary to monitor the separation of steel reinforcement from concrete. The integrity of the reinforced concrete structure must be preserved, therefore, methods and means of non-destructive testing play an important role in the operation of reinforced concrete products. If the method of impedance spectroscopy is often used to determine delamination [1], then to determine such parameters as the diameter of reinforcing bars and the distance from the reinforcing mesh to the surface of a reinforced concrete product, the greatest success has been achieved using the magnetic method of non-destructive testing. The essence of the method is reduced to the formation of a primary magnetic field of a certain configuration (or a set of configurations), registration of the spatial distribution of the measured parameter and, on this basis, reconstruction of the initial three-dimensional distribution of ferromagnetic objects in the controlled area. The thickness of the concrete cover and the location of the steel reinforcement in the structure are usually determined based on the experimentally established relationship between the parameters of the stray field and the specified controlled parameters of the structure [2]. To determine the distance, you need to know the value of the stray field, and the diameter of the reinforcement can be calculated from the magnitude of the stray field only with a known distance to the measuring point. The magnitude of the measured stray field will be influenced by both the magnetic properties of the armature and the magnitude of the magnetizing field. 
Today, there are a number of ready-made devices that implement the magnetic method of nondestructive testing of reinforcement in concrete (for example, IPA-MG4.1 (SKB Stroypribor), Profoscope (Proceq, Switzerland), Ferroscan PS 250 (Hilti, Liechtenstein)). Despite the great variety, all existing products and technologies solve the problem with approximately the same accuracy. In [3], an experimental study of the magnitude of the error that accompanies the magnetic control method in determining the diameter of reinforcement and a protective layer of concrete using IPA-MG4.1 and Profoscope was carried out. As a result, it was concluded that the investigated devices determine the depth of the reinforcement with an error of $5-10 \%$, and the diameter of the reinforcing bars with an error of $15-30 \%$. It should be noted that the experimental results were obtained under conditions close to ideal: the depth of occurrence was determined with a known diameter of the reinforcement; the diameter was determined at a known burial depth; there are no adjacent parallel and perpendicular reinforcing bars. In real studies, it is almost impossible to achieve these conditions, therefore, the error in determining the depth of occurrence can be $50-60 \%$, and the diameter of the rods - up to $100 \%$.

Earlier in [4], a dipole model of local magnetization of reinforcement was proposed, which makes it possible to significantly increase the reliability of the application of the magnetic method for determining independently of each other the distance to the reinforcement and the diameter of the reinforcement. To determine the distance, the amplitude of the stray magnetic field from the locally magnetized section of the armature and the gradient of the magnetic field were measured. A permanent magnet acted as a magnetizing system; magnetic field sensors were located on the side of the magnet. It was shown that the magnitude of the magnetizing field and the diameter of the reinforcement do not affect the determination of the distance. Also, a scientific position was formulated that the diameter of the reinforcement can be determined regardless of the magnetic properties of the reinforcement, provided that such fields are created in the material, close to the coercive force, at which the magnetic permeability of the reinforcing bar tends to its maximum value $\left(\mu(H) \rightarrow \mu_{\max }\right)$. The purpose of this work is to confirm the advanced scientific position, as well as to study the influence on the measured field of the geometric parameters of the magnetized section of the reinforcement and its magnetic properties.

\section{Main part}

The magnetic induction in the magnetized section of the reinforcement, taking into account the demagnetization coefficient, can be calculated by the formula:

$$
B=\mu_{0} \mu_{\text {body }} H_{e}=\frac{\mu_{0} H_{e}}{\frac{1}{\mu(H)}+N\left[\frac{\mu(H)-1)}{\mu(H)}\right]},
$$

where $H_{e}$ - field created by a permanent magnet dipole:

$$
H_{e}=\frac{2 \cdot q_{M} \cdot \frac{1}{2} L_{M}}{\left(z^{2}+\frac{1}{4} L_{M}^{2}\right)^{\frac{3}{2}}}
$$

Provided that $\mu(H) \rightarrow \mu_{\max }$, that is $\mu_{\max } \gg 1$, then:

$$
B \approx \frac{\mu_{0} H_{e}}{\frac{1}{\mu(H)}+N}
$$


If we assume that the magnetized section of the reinforcement is an ellipsoid, then the demagnetizing coefficient can be easily estimated as the ratio of the diameter of the reinforcement to the length of the magnetized section.

$$
N \approx \frac{D}{L} \cdot \alpha
$$

Then, if the length of the magnetized section of the reinforcement does not exceed ten of its diameters, then $N \approx 0.25 \ldots 0.33$ and $N \gg \frac{1}{\mu(H)}$, those:

$$
B \approx \frac{\mu_{0} H_{e}}{N}
$$

In reality, the shape of the locally magnetized section of the reinforcement can differ significantly from the ellipsoid, therefore, $N$ and $\frac{1}{\mu(H)}$ may be comparable in magnitude and it is necessary to be guided by formula (3). In addition, the length of the magnetized section varies depending on the length of the magnet and the distance from the magnet to the reinforcing bar, therefore, using formula (4), only a qualitative estimate can be obtained. More precisely, the demagnetizing coefficient should be determined based on the results of numerical simulation by the finite element method.

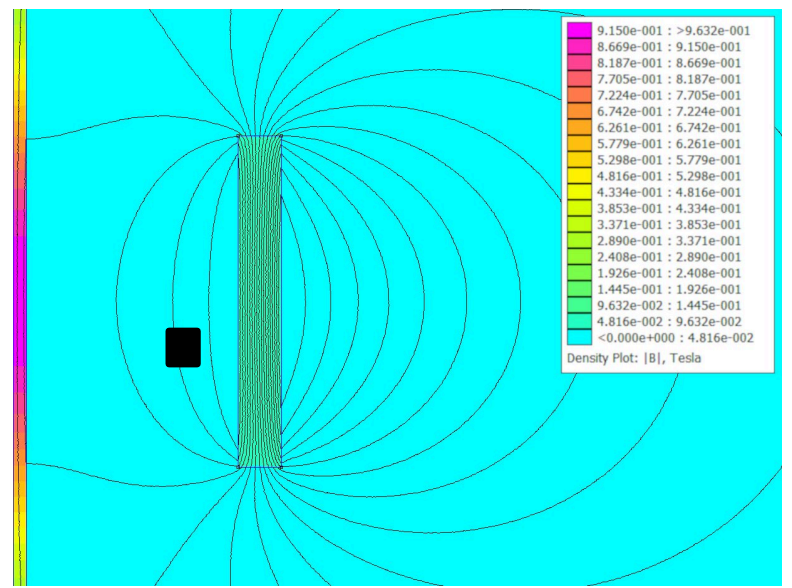

Fig. 1. Distribution of magnetic induction obtained in the FEMM software package 4.2

This work contains the results of a series of calculations in the FEMM 4.2 software package. The response of a reinforcing bar to an external magnetic field created by a permanent magnet is considered for various reinforcement diameters of 8,12 and $16 \mathrm{~mm}$ and a range of $\mu=100 \ldots 5000$. The magnetic field strength was determined at a point near the bottom surface of a permanent magnet (black square in Fig. 1): at this point, the location of the magnetic field sensor in a real device that implements this method is assumed. For example, in this Fig. 1 shows the distribution of magnetic induction when solving an axisymmetric problem for a reinforcing bar with a diameter of $8 \mathrm{~mm}$ and an annular magnet with a section of $100 \times 12.5 \mathrm{~mm}$ and $\mu=1000$. The calculation results fully correspond to the planar problem with the magnet dimensions $100 \times 100 \times 12.5 \mathrm{~mm}$, since the characteristics of the permanent magnet were selected based on the correspondence of the volumes of the sector of the ring magnet and the magnet in the form of a parallelepiped. The volume of the latter is $0.125 \mathrm{dm}^{3}$ (mass $1 \mathrm{~kg}$ ), while the volume of the ring magnet is 10 times 
larger, therefore, in the calculation, the coercive force of the magnet was reduced 10 times compared to the nominal value. In this case, the distributions of the magnetic field from the sector of the ring magnet, which occupies $1 / 10$ of its total volume, and the magnet in the form of a parallelepiped, will be similar.

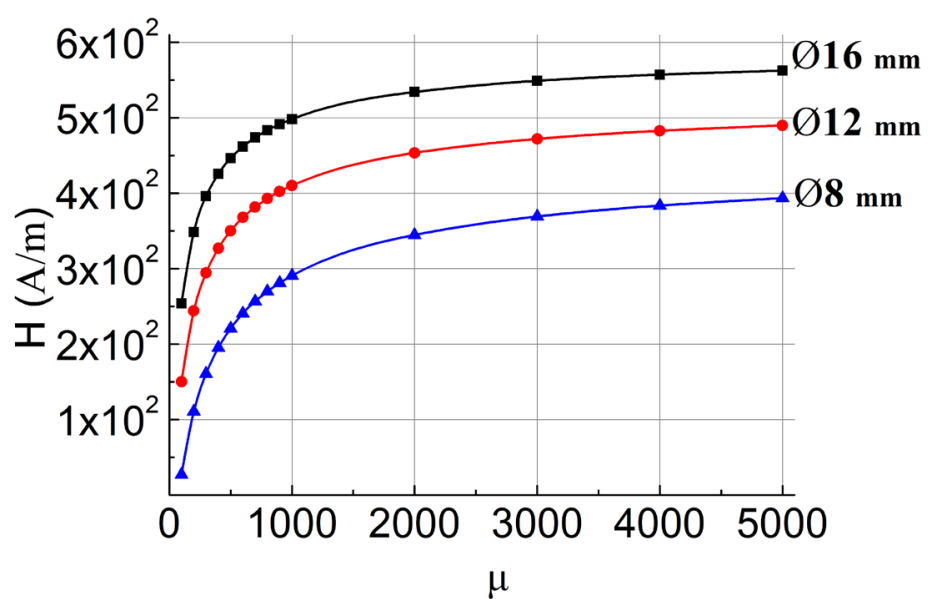

Fig. 2. Magnetic field strength of the response of the reinforcing bar to an external magnetizing field, depending on the magnetic properties of the reinforcing bar.

Sectional dimensions of the ring magnet $100 \times 12.5 \mathrm{~mm}$

The results of calculating the strength of the magnetic field created by the magnetized section of the reinforcement, depending on the magnetic properties of the reinforcing bar, are shown in Fig. 2. The cross-sectional dimensions of the ring magnet are $100 \mathrm{x} 12.5 \mathrm{~mm}$. It can be seen from the figure that there is an interval of $\mu$ values, at which it is possible to unambiguously determine the diameter of the reinforcement at a known magnetic field strength - from 2000 to 5000. In this interval, the graphs have a linear character and the unique correspondence of the $H$ value to a single $\mu$ value on one of the graphs for different $d$.

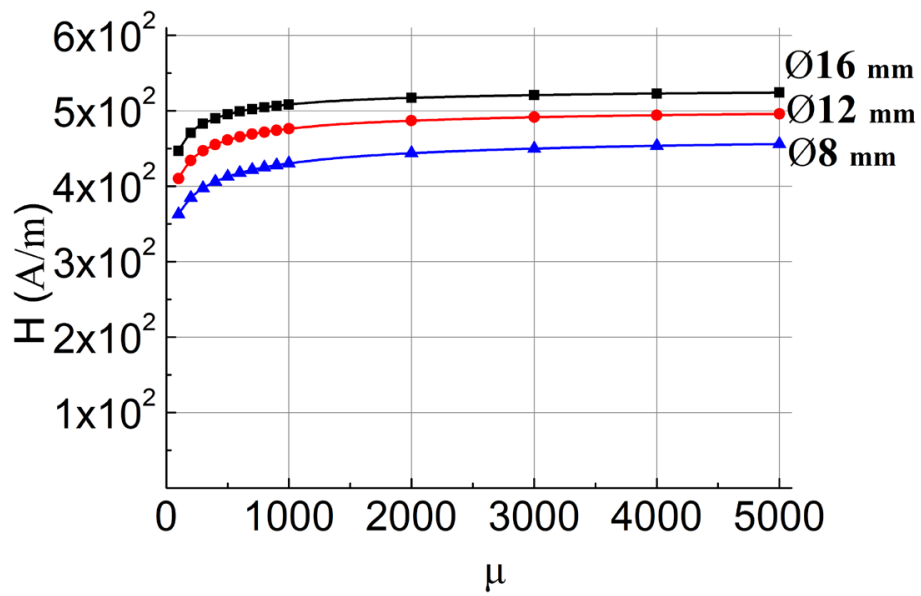

Fig. 3. Magnetic field strength of the response of the reinforcing bar to an external magnetizing field, depending on the magnetic properties of the reinforcing bar.

Sectional dimensions of ring magnet $50 \times 25 \mathrm{~mm}$ 
Structural steels from which reinforcing bars are made, according to national standards, can have $\mu_{\max }$ values from 1500 to 3500 , therefore, it is necessary to expand the range of unique definitions of reinforcement. This can be done, according to expressions $(4,5)$, by changing (downward) the length of the magnetized section of the reinforcement. Such a case was also considered, the results are presented in Fig. 3. The cross section of the ring magnet did not change, and the dimensions were $50 \times 25 \mathrm{~mm}$. Analyzing the graphs, we can say with confidence that the minimum of the interval for the unambiguous determination of $d$ has expanded to $\mu=1000$, therefore the proposed method can be applied to all structural steels with the properties stated above

\section{Conclusion}

1) In the work, using a series of numerical experiments, it was proved that due to only one measurement of the absolute value of the magnetic field strength, it is possible to determine the diameter of the reinforcing bars with great accuracy, regardless of the magnetic properties of the material from which they are made.

2) Expansion of the range in which the diameter of the reinforcement is determined unambiguously is possible by reducing the length of the magnetized section of the reinforcing bar. This is achieved by changing the geometric parameters of the magnetizing system.

\section{Acknowledgments}

The work was performed in accordance with the theme "Diagnostics" No. AAAA-A18118020690196-3.

\section{References}

[1] S. Hong, R. Harichandran, Sensors to Monitor CFRP/Concrete Bond in Beams Using Electrochemical Impedance Spectroscopy, J COMPOS CONSTR. 9 (2005) 515-523. https://doi.org/10.1061/(ASCE)1090-0268(2005)9:6(515)

[2] GOST 22904-93. Konstrukcii zhelezobetonnye. Magnitnyj metod opredelenija tolshhiny zashhitnogo sloja betona i raspolozhenija armatury.

[3] Vasil'ev A.I., Ocenka korrozionnogo iznosa rabochej armatury v balkah proletnyh stroenij avtodorozhnyh mostov, Beton i zhelezobeton. 2 (2000) 20-23.

[4] Gobov Y.L., Mikhailov A.V., Smorodinskii Y.G., Magnetic method for nondestructive testing of rebar in concrete, Russian journal of nondestructive testing. 54 (2018) 871-876. https://doi.org/10.1134/S1061830918120057 